\title{
Results of the ONTARGET and TRANSCEND studies: an update and discussion
}

\author{
David Fitchett \\ St Michael's Hospital, and University \\ of Toronto, Ontario, Canada
}

\begin{abstract}
The renin angiotensin aldosterone system (RAAS) plays an important role in the pathophysiology of cardiovascular disease. Angiotensin converting enzyme inhibitors (ACEi) have proven benefit in reducing cardiovascular events in patients at high risk. Angiotensin receptor blockers (ARB) have been demonstrated to have benefit in the management of heart failure and to be non-inferior to ACEi in patients with left ventricular dysfunction after a myocardial infarction (MI). Yet until now, there has been no trial to support the use of the ARB for vascular protection. The ONTARGET study showed that the ARB telmisartan conserved $95 \%$ of the vascular protective properties of the ACEi ramipril, given at similar doses to a similar patient group as had been previously shownin the HOPE study to benefit from ACE inhibition with ramipril. The TRANSCEND study in a similar population of patients who were intolerant of ACEi despite the primary endpoint being neutral, showed a trend to a benefit for the combined secondary endpoint of cardiovascular death, MI and stroke, with excellent tolerance of the ARB. The reasons for neutral result of the TRANSCEND study result include an underpowered study, and pre-treatment with a RAAS inhibitor in a high proportion of patients. These studies indicate that an ARB can be used for vascular protection in high risk individuals in the place of an ACEi. However ACEi will probably remain the first choice due to the greater body of supportive evidence.
\end{abstract}

Keywords: angiotensin receptor blocker, angiotensin converting enzyme inhibitor, vascular protection

\section{Introduction}

Modulation of the renin angiotensin aldosterone system (RAAS) with angiotensin converting enzyme inhibitors (ACEi) reduces cardiovascular events in individuals at high risk. ${ }^{1}$ Clinical trials in hypertension and heart failure suggested the angiotensin receptor blockers (ARB) might have the vascular protective benefits of the ACEi with less adverse effects. However approximately $20 \%$ of patients, especially women and Asians, are unable to take an ACEi due to adverse effects such as cough, hypotensive symptoms, and angioneurotic edema. Consequently, an angiotensin receptor blocking agent (ARB) could be an alternative treatment to ACEi for vascular protection, with better patient tolerability.

The results of the ONTARGET ${ }^{2}$ and TRANSCEND $^{3}$ studies, the largest clinical trial program ever conducted with an angiotensin receptor blocker, were recently reported. These trials examined strategies for cardiovascular risk reduction in high risk patients aged $>55$ years old, with coronary, cerebrovascular disease, peripheral vascular disease or diabetes with target organ damage. ONTARGET compared the ARB telmisartan alone, or in combination with the ACEi ramipril, with ramipril treatment, for the protection against major cardiovascular events such as cardiovascular death, myocardial infarction (MI), stroke, or hospitalization for heart failure. TRANSCEND compared telmisartan 
to placebo in patients who were intolerant of ACEi. The aims of the ONTARGET/TRANSCEND program were to learn whether adding the ARB telmisartan to proven therapy with an ACEi would further improve cardiovascular outcomes, and to establish the benefits of the ARB in this high risk population.

\section{Vascular protection and RAAS modulation}

Activation of the RAAS system is implicated in a range of cardiovascular and renal pathophysiology: ${ }^{4}$ the development of atherosclerosis, the complications of atherosclerosis such as acute $\mathrm{MI}$, adverse myocardial remodeling and the development of heart failure, the development of systemic hypertension, and the development of progressive renal dysfunction. Angiotensin II mediated stimulation of the AT1 receptor increases arterial pressure, promotes oxidative stress, stimulates an inflammatory response, and adversely alters the balance between the thrombotic and fibrinolytic state (Figure 1). Experimental evidence supports the hypothesis that both ACEi and the ARB classes of RAAS modulators have properties that may reduce the development of atherosclerosis and its complications. However differences between ACEi and ARB responses are observed, such that the ARBs might be pro-thrombotic by stimulating PAI-1 synthesis ${ }^{5}$ and and encourage plaque rupture by enhancing MMP-1 activity. ${ }^{6}$

\section{Angiotensin converting enzyme inhibition and vascular protection}

The SOLVD ${ }^{7}$ and $\mathrm{SAVE}^{8,9}$ studies were designed to test the hypothesis that RAAS modulation with an ACEi would slow the progression and development of heart failure. A surprising finding of both studies was a $20 \%$ reduction of the incidence of MI. These observations lead to the HOPE study, ${ }^{10}$ in which high dose ACEi with ramipril $10 \mathrm{mg}$ daily reduced the risk of MI by $20 \%$, stroke by $32 \%$, and cardiovascular mortality by $26 \%$ in patients at high risk for cardiovascular events but without heart failure or a low left ventricular ejection fraction. The EUROPA study ${ }^{11}$ supported the observations of the HOPE study. Perindopril $8 \mathrm{mg}$ daily in patients with coronary artery disease, reduced the endpoint of cardiovascular mortality, non-fatal MI, and cardiac arrest by $20 \%$. The PEACE study ${ }^{12}$ failed to show a benefit from treatment with trandolapril $4 \mathrm{mg}$ daily in patients with coronary artery disease. The study population was at low risk of cardiovascular events and the trial underpowered to show a benefit from the ACEi treatment. Coronary revascularization was the major contributor to the combined endpoint and occurred at similar rates in both treatment arms. In contemporary North
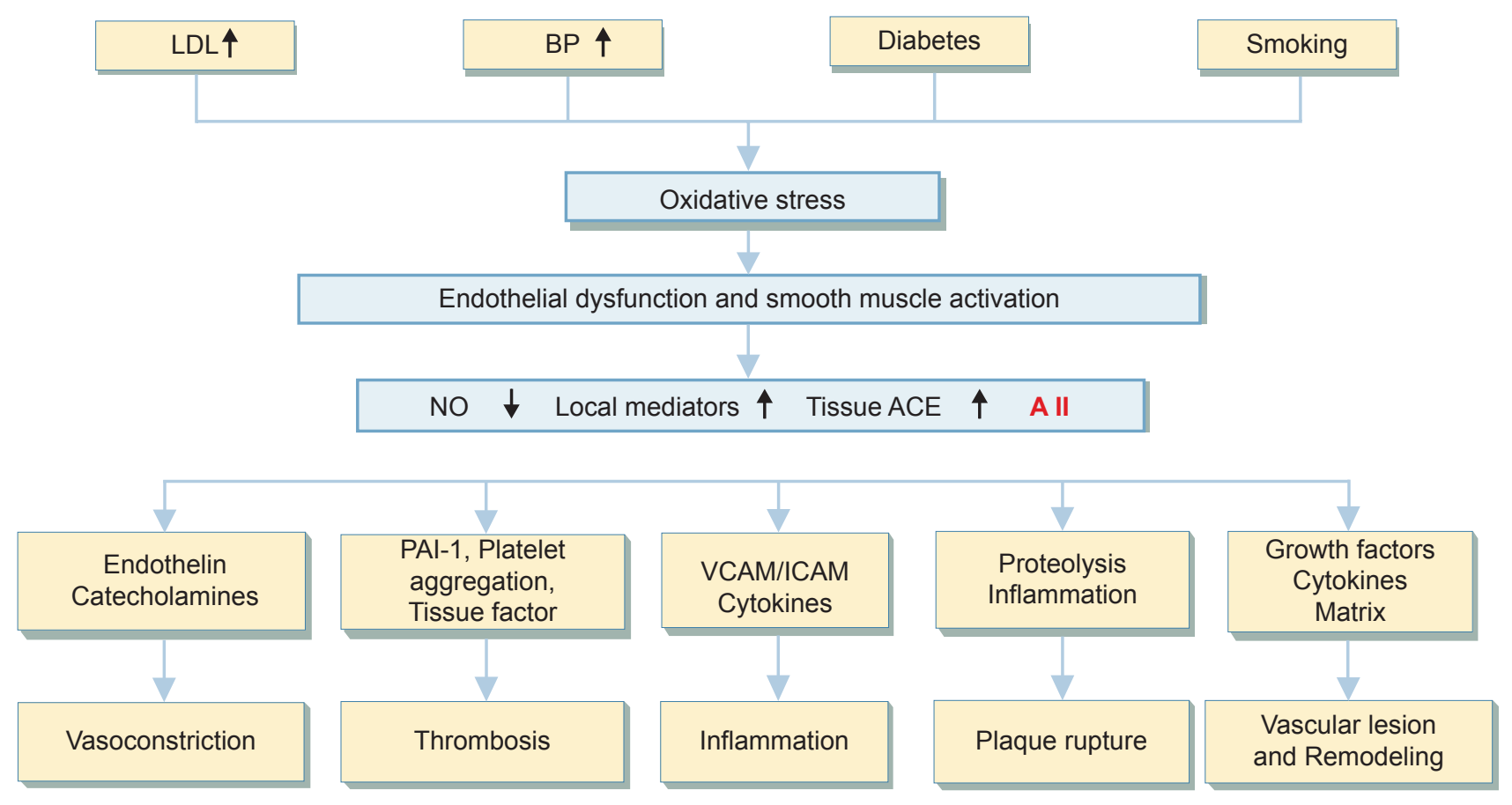

Figure I The role of the renin angiotensin aldosterone system in vascular disease. Angiotensin II (All) is an important mediator of vascular damage that promotes the development of atherothrombosis and the complications of atherosclerosis. 
American practice, aggressive coronary revascularization is probably a non-modifiable endpoint, as it is widely used independent of ischemia driven indications.

A combined analysis ${ }^{13}$ of the HOPE, EUROPA and PEACE studies showed that ACEi significantly reduced all-cause mortality $(7.8$ vs $8.9 \% \mathrm{p}=0.0004)$, cardiovascular mortality (4.3 vs 5.2\% p = 0.0002), non-fatal MI (5.3 vs 6.4\% $\mathrm{p}=0.0001)$, stroke $(2.2 \mathrm{vs} 2.8 \% \mathrm{p}=0.0004)$, coronary artery bypass surgery ( $6.0 \mathrm{vs} 6.9 \%)$, but not percutaneous coronary intervention ( 7.4 vs $7.6 \% \mathrm{p}=0.48)$. Similar reductions of cardiovascular mortality and MI are observed in the five trials in patients with heart failure or left ventricular systolic dysfunction. ${ }^{14}$

\section{ARBs and vascular protection: pre-ONTARGET/TRANSCEND}

Unlike the ACEi studies, most of which compared the ACEi with placebo, the ARBs have been compared with an active comparator. In heart failure trials, the comparator was usually an ACEi, whereas in hypertension trials the ARB was compared with other medication strategies. Placebo-controlled clinical trials with evaluation of clinical outcomes are either in ACEi-intolerant patients (CHARM alternative ${ }^{15,16}$ ) or in patients with diabetic nephropathy (RENAAL ${ }^{17}$ and IDNT $^{18}$ ).

The CHARM study ${ }^{19}$ showed that the addition of the ARB candesartan to standard treatment, that included an ACEi reduced cardiovascular deaths and hospital admissions for heart failure. The combination of candesartan with an $\mathrm{ACEi}^{20}$ was associated with a $17 \%$ reduction of both cardiovascular mortality and admission to hospital for heart failure compared to those receiving only an ACEi. Patients receiving candesartan and an ACEi had a 23\% (95\% CI 2\%-40\%) reduction of non-fatal $\mathrm{MI}^{21}$ and a $12 \%$ (95\% CI 13\%-21\%) reduction of cardiovascular death. Yet in the CHARM Alternative trial, ${ }^{15,16}$ where candesartan was compared to placebo in patients intolerant of ACEi, the incidence of MI increased by $52 \%$, but cardiovascular death was $20 \%$ less $(p=0.02$ after covariate adjustment).

ARBs are effective and well tolerated antihypertensive agents. The LIFE study ${ }^{22}$ in older patients with moderately severe hypertension and left ventricular hypertrophy, showed the ARB losartan when compared to atenolol, resulted in a greater reduction of the combined end-point of cardiovascular death, MI or stroke after equal blood pressure control. In patients with diabetes, losartan reduced cardiovascular and total mortality, but not MI. In the VALUE study, ${ }^{23}$ valsartan was not as effective an antihypertensive agent as amlodipine, yet there was no difference in the primary combined endpoint of cardiovascular mortality and morbidity. Yet, the valsartan-treated patients had a $19 \%$ relative increase in MI $(\mathrm{p}=0.02)$ and a $15 \%(\mathrm{p}=0.08)$ increase in stroke compared to individuals receiving amlodipine.

ACEi in patients following MI reduce the risk of death by $26 \%$, heart failure admissions by $27 \%$, and recurrent MI by $20 \% .^{24}$ The ARB valsartan in the VALIANT study ${ }^{25}$ was shown to be non-inferior to captopril for the reduction of all-cause mortality in patients with heart failure or left ventricular ejection fraction $<40 \%$. In this study MI occurred as frequently in valsartan as in captopril treated patients. Yet a discussion paper ${ }^{26}$ based on a meta-analysis of over 50,000 patients, suggested that ARBs might increase the incidence of MI. An alternative explanation to this hypothesis is that the comparators in these trials (including ACEi and beta-adrenergic blockers) were more effective than an ARB for the reduction of coronary events.

The nephropathy studies RENAAL ${ }^{17}$ and IDNT $^{18}$ showed that ARBs compared to placebo successfully slowed the progression of renal disease. Yet in these high risk patients, it is surprising that ARBs did not reduce vascular endpoints. It is likely these trials, primarily designed to examine renal endpoints, were underpowered to answer questions about cardiovascular events.

\section{ONTARGET/TRANSCEND study design $^{27}$}

The Ongoing Telmisartan Alone and in Combination with Ramipril Global Endpoint Trial (ONTARGET) ${ }^{2}$ and Telmisartan Randomized Assessment Study in ACE Intolerant Subjects with Cardiovascular Disease (TRANSCEND) ${ }^{3}$ studies examined the role of ARBs when used alone or in combination with an ACEi in high risk individuals with controlled hypertension (Figure 2). The design of the ONTARGET/TRANSCEND studies closely resembled that of the HOPE study, knowing that ramipril in this population was beneficial and to allow comparisons of the outcomes of the two trials. The primary objective of ONTARGET was to determine whether the combination of telmisartan and ramipril was more effective than ramipril alone and to assess whether telmisartan alone was at least as effective as ramipril. TRANSCEND was a placebo-controlled study to determine whether telmisartan reduces vascular endpoints compared to placebo in patients intolerant of ACEi.

The primary endpoint for both ONTARGET and TRANSCEND was the combination of cardiovascular death, non-fatal MI, stroke, or hospitalization for heart failure. 


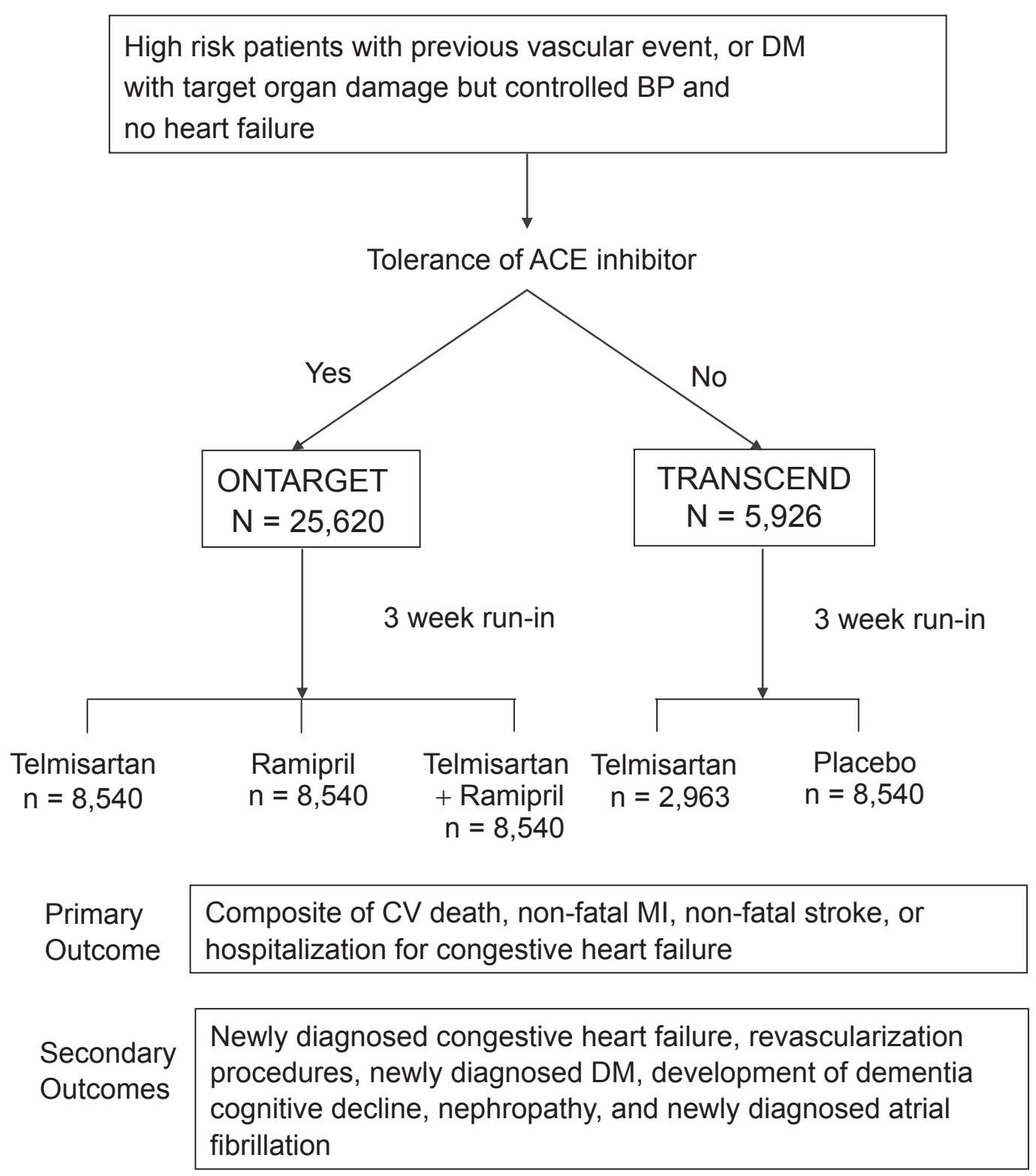

Figure 2 Study design and outcomes of ONTARGET and TRANSCEND studies.

Both studies included patients at high risk of coronary, peripheral or cerebrovascular events. Recruitment from 730 centers in 40 countries for ONTARGET $(n=25,620)$ was completed in July 2003, and TRANSCEND $(n=6000)$ in May 2004. The planned follow-up period was 3.5 to 5.5 years. The study was designed to have a $93 \%$ power of showing a $13 \%$ superiority of telmisartan plus ramipril vs ramipril alone. In addition the study had an $89 \%$ power of showing the non inferiority of telmisartan compared to captopril whilst ensuring telmisartan has at least $50 \%$ of the ramipril effect at the upper 95 th $\%$ confidence limit. In TRANSCEND with 3000 patients in each group, the study had a 94\% power to show a $19 \%$ superiority of telmisartan over placebo, assuming an event rate of 5.1\%/year, a recruitment period of 2 years and a maximum follow-up of 5.5 years.

\section{Baseline characteristics in ONTARGET and TRANSCEND}

The age of the patients in ONTARGET (66.4 years) and TRANSCEND (66.9 years) was slightly greater than in HOPE (65.9 years). Diabetes was present with a similar prevalence in ONTARGET (37.3\%) and TRANSCEND (35.4\%) as in HOPE (38.3\%). ACEi (57.5\%) and statin (61\%) usage prior to enrollment was considerably greater than reported in HOPE (ACEi 11.6\%, statins 28.9\%). It is notable that there was a baseline 56\% ACEi usage in TRANSCEND, in patients 
supposedly intolerant to ACEi. The TRANSCEND population had several baseline differences from both ONTARGET and HOPE. In TRANSCEND there were a higher proportion of female patients $(42.9 \%)$ than in ONTARGET $(26.7 \%)$ or HOPE (26.7\%).

\section{Results of ONTARGET}

Differences in blood pressure between the treatment groups during the trial were modest. The average difference of blood pressure for the telmisartan-treated groups (compared to ramipril) was $0.9 / 0.6 \mathrm{mmHg}$, and for telmisartan + ramipril 2.4/1.4 mmHg.

After a median follow-up of 56 months the incidence of the primary outcome of cardiovascular death, non-fatal MI, stroke, or the development of heart failure did not differ significantly between either the telmisartan + ramipril or the telmisartan groups compared to the ramipril group. The confidence intervals of the relative risk of the telmisartan vs ramipril comparison were within the pre-specified boundaries to show that telmisartan was not inferior to ramipril, and had conserved $95 \%$ of the efficacy of ramipril (Figure 3 ). Consequently ONTARGET shows that telmisartan was as effective as ramipril, and well tolerated.

The combination of telmisartan and ramipril did not result in any improved outcome beyond that achieved by ramipril alone. Yet the combined treatment resulted in more hypotension, syncope, renal dysfunction, hyperkalemia, and a trend towards a greater risk of renal dysfunction requiring dialysis. A subsequent analysis ${ }^{28}$ of the renal outcomes of ONTARGET showed that the combination of telmisartan and ramipril increased the renal endpoints of dialysis and doubling of creatinine and death compared to treatment with the individual agents: ramipril $13.4 \%$, telmisartan $13.5 \%$, ramipril + telmisartan 14.5\% RR $1.09(\mathrm{p}=0.037)$ and dialysis and doubling of creatinine ramipril $2.03 \%$, telmisartan $2.21 \%$, ramipril + telmisartan 2.49\% RR 1.24, $(\mathrm{p}=0.038)$. Many of the dialysis events were for acute dialysis, probably resulting from acute renal failure due to excessive blood pressure reduction in patients with atherosclerotic renal artery disease. For the 700 patients with diabetic nephropathy, the combined telmisartan + ramipril treatment resulted in a non-significant $8 \%$ reduction of the combined endpoint of death, doubling of creatinine, or need for dialysis. Consequently, there is no benefit of ACEi + ARB combination therapy in any group of patients included in the ONTARGET study, beyond a reduction of albuminuria.

\section{TRANSCEND}

The TRANSCEND study ${ }^{3}$ comparing telmisartan and placebo in high risk patients intolerant of ACEi failed to show a clear benefit of ARB therapy. Blood pressure was reduced $4 / 2 \mathrm{mmHg}$ by telmisartan. The primary end-point of death, MI, stroke or admission to hospital with heart failure occurred in $15.7 \%$ of patients receiving telmisartan and $17.0 \%$ receiving placebo (HR $0.92, p=0.216$ ). The secondary HOPE endpoint of death, MI, stroke (Figure 3) appeared to be reduced ( $13 \%$ vs $14.8 \%$, HR $0.87, \mathrm{p}=0.048)$. However after adjustment for multiple comparisons, the difference was non-significant $(\mathrm{p}=0.068)$. Telmisartan was well tolerated in patients reported to be previously intolerant of ACEi, and fewer patients discontinued telmisartan than discontinued placebo treatment. Hypotensive symptoms were the commonest, yet very infrequent adverse event (telmisartan $0.98 \%$ vs placebo $0.54 \%$ ) resulting in permanent discontinuation of treatment. In addition the safety of telmisartan was good in the 377 patients who had previously had severe reactions to an ACEi.

The Prevention Regimen for Effectively Avoiding Second Strokes (PRoFESS) Study ${ }^{29}$ of more than 20,000 patients, compared the effect of telmisartan $80 \mathrm{mg}$ daily with placebo in the secondary prevention of cardiovascular events including a further stroke. Despite a blood pressure reduction of 3.8/2.0 $\mathrm{mmHg}$ there was no reduction of the incidence of subsequent stroke or major cardiovascular events.

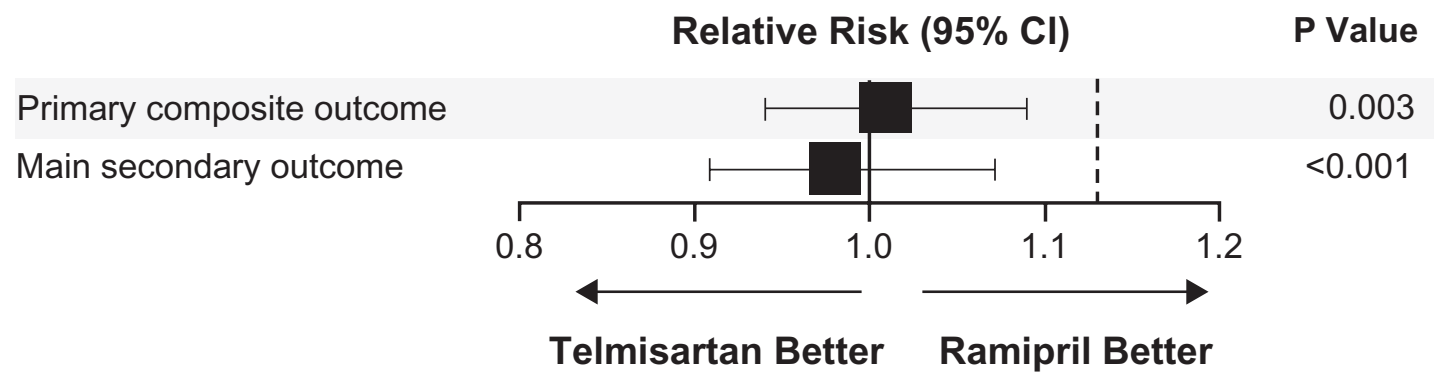

Figure 3 Relative risk for primary outcome (cardiovascular death, MI, stroke and hospitalization for heart failure) and secondary HOPE study outcome (cardiovascular death, $\mathrm{MI}$ or stroke). The confidence interval is well within the predefined boundary for non-inferiority of telmisartan compared with ramipril. 
Outcomes appeared to be improved for events that occurred after 6 months of treatment. In a pre-specified combined analysis of TRANSCEND and PRoFESS with more than 26,000 patients, benefit from telmisartan was seen after 6 months treatment (cardiovascular death, MI, stroke, hospitalization for heart failure OR 0.86 (95\% CI 0.80-0.94). However there was little or no benefit in the first 6 months after randomization (OR 1.12 95\% CI 0.99-1.27). It is therefore possible that more prolonged treatment with telmisartan could have resulted in a greater benefit. In TRANSCEND, the mean duration of treatment to the occurrence of a vascular event is only $2 \frac{1}{2}$ years: a short time frame by comparison of the time taken for blood pressure lowering or lipid-lowering agents to show significant benefit.

\section{Discussion}

\section{$A R B=A C E i$ ?}

The vascular protective property of ACEi has been shown in clinical trials and subsequent meta-analyses. Studies with ARBs in patients with hypertension, heart failure and renal disease have yielded conflicting results. The ONTARGET study in patients at high risk of cardiovascular events showed that telmisartan was non-inferior, as defined by pre-specified boundaries, to the ACEi ramipril given at the same dose as had been proven to be beneficial in the HOPE study. The study showed that telmisartan conserved 95\% (95\% CI 83.2-106.3) of the benefits of ramipril assessed by the primary composite outcome and 105\% (95\% CI 91.6-119.0) of the benefits assessed by the secondary outcome of cardiovascular death, MI and stroke. These benefits were observed in a high risk population with Death/MI/Stroke event rates similar to those observed in the index HOPE study (cardiovascular/year rates: ONTARGET 3.0\%/year, HOPE 2.8\%/year). Consequently the equivalence of telmisartan to the ACEi ramipril for clinical application has been conclusively demonstrated. The ONTARGET study has raised several questions about the generalizabilty of the findings. Can we assume that all ARBs can be used to replace an ACEi? Does telmisartan have any special properties? Telmisartan has a long biological half-life, and has PPAR agonist properties in addition to those of an AT1 receptor blocker. Whether PPAR agonist activity is beneficial or harmful in this situation is unknown. Other ARBs in heart failure trials have shown equal vascular protection when added to treatment that included an ACEi (CHARM overall, ${ }^{20}$

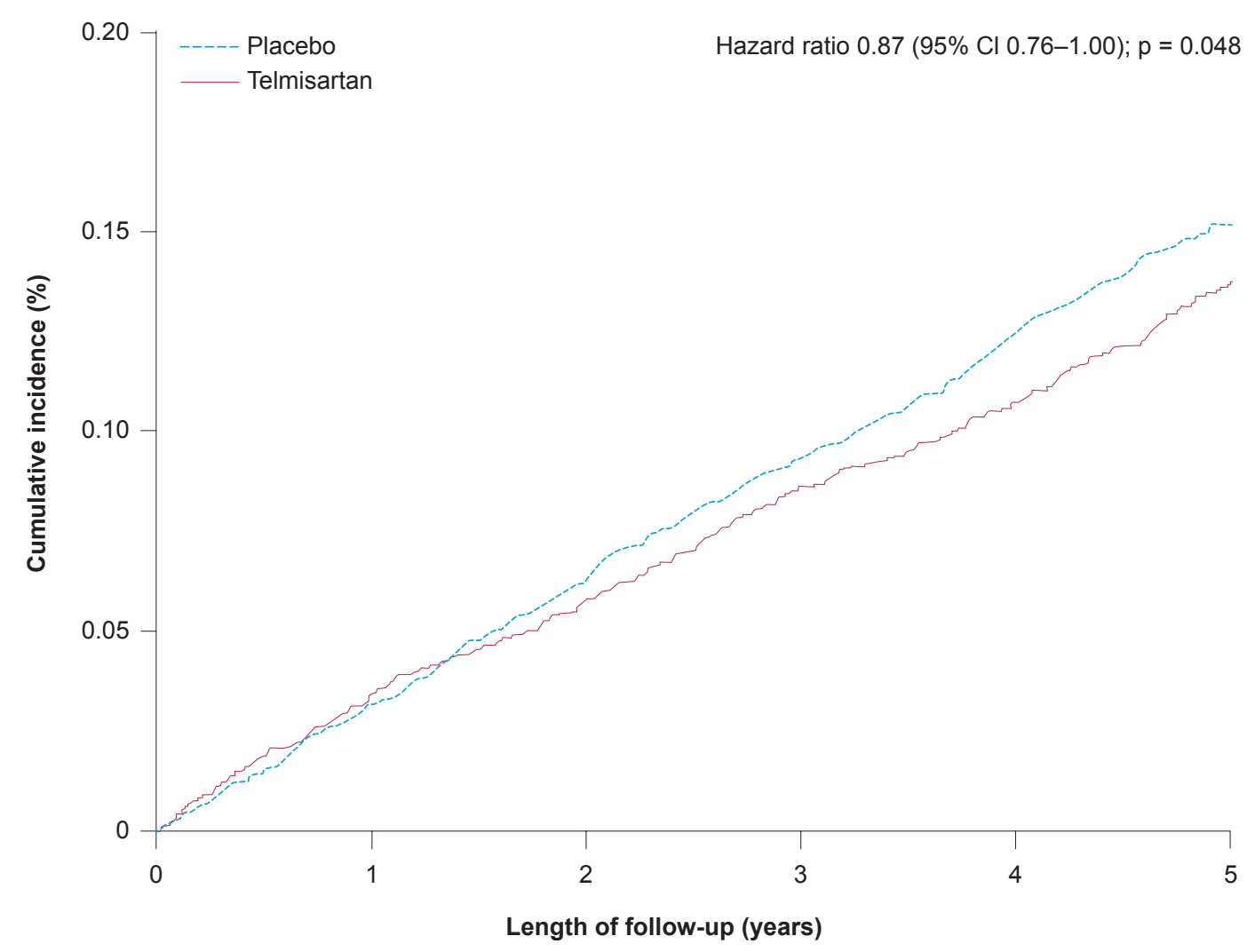

Figure 4 TRANSCEND study comparing telmisartan $80 \mathrm{mg}$ daily with placebo in high risk patients. Kaplan-Meier Curves for the secondary outcome of cardiovascular death, MI or stroke (HOPE study outcome). After statistical correction for multiple comparisons and overlap with the primary endpoint, the difference was not statistically significant $(p=0.68)$. 
or compared to an ACEi (VALIANT ${ }^{25}$ ). Yet the same ARBs in other situations when compared to placebo have been associated with an increased risk for MI (CHARM alternative ${ }^{15,16}$ in ACE intolerant patients and VALUE ${ }^{23}$ in high vascular risk patients with hypertension. So there is some uncertainty whether other ARBs would have been non-inferior to ramipril if they had been tested in an ONTARGET-like trial. Furthermore we do not know the optimal dose of other agents. However, the dose of telmisartan for ONTARGET was chosen as that known to have an effective antihypertensive action: the same reasoning could be applied to select the dose of other ARBs for vascular protection.

\section{Why no additional benefit from ACEi + ARB?}

The combination of ramipril and telmisartan resulted in no additional benefit beyond that achieved with either drug alone. Furthermore the combination therapy was associated with an increased incidence of adverse effects including those related to symptomatic hypotension, a deterioration of renal function, and hyperkalemia. The ACEi/ARB combination did reduce blood pressure more than the single agent. As a part of the vascular protective benefit of ACEi had previously been attributed to blood pressure reduction, it was surprising to see no additional benefit with average BP 2.4/1.4 mmHg lower in the combined arm compared to ramipril treated patients. Perhaps maximal vascular protection had been achieved with ACEi or ARB alone, and a further reduction of BP and RAAS activity had no additional benefit.

\section{Is there an indication for combined ACEi + ARB?}

The combination of ACEi and ARB has not been proven to be an effective antihypertensive strategy and is not recommended by the Canadian Hypertensive Education Program (CHEP) unless there are other compelling reasons for combination therapy such as with heart failure or renal disease. The ONTARGET study shows that in the population studied there is no reason to combine ACEi and ARB. For patients with severe heart failure the CHARM Overall ${ }^{20}$ study with candesartan appears to support combined therapy. However the choice and dose of ACEi was not specified and similar risk reductions were seen whether or not the patient was taking an ACEi. When the ACEi and dose were determined by the

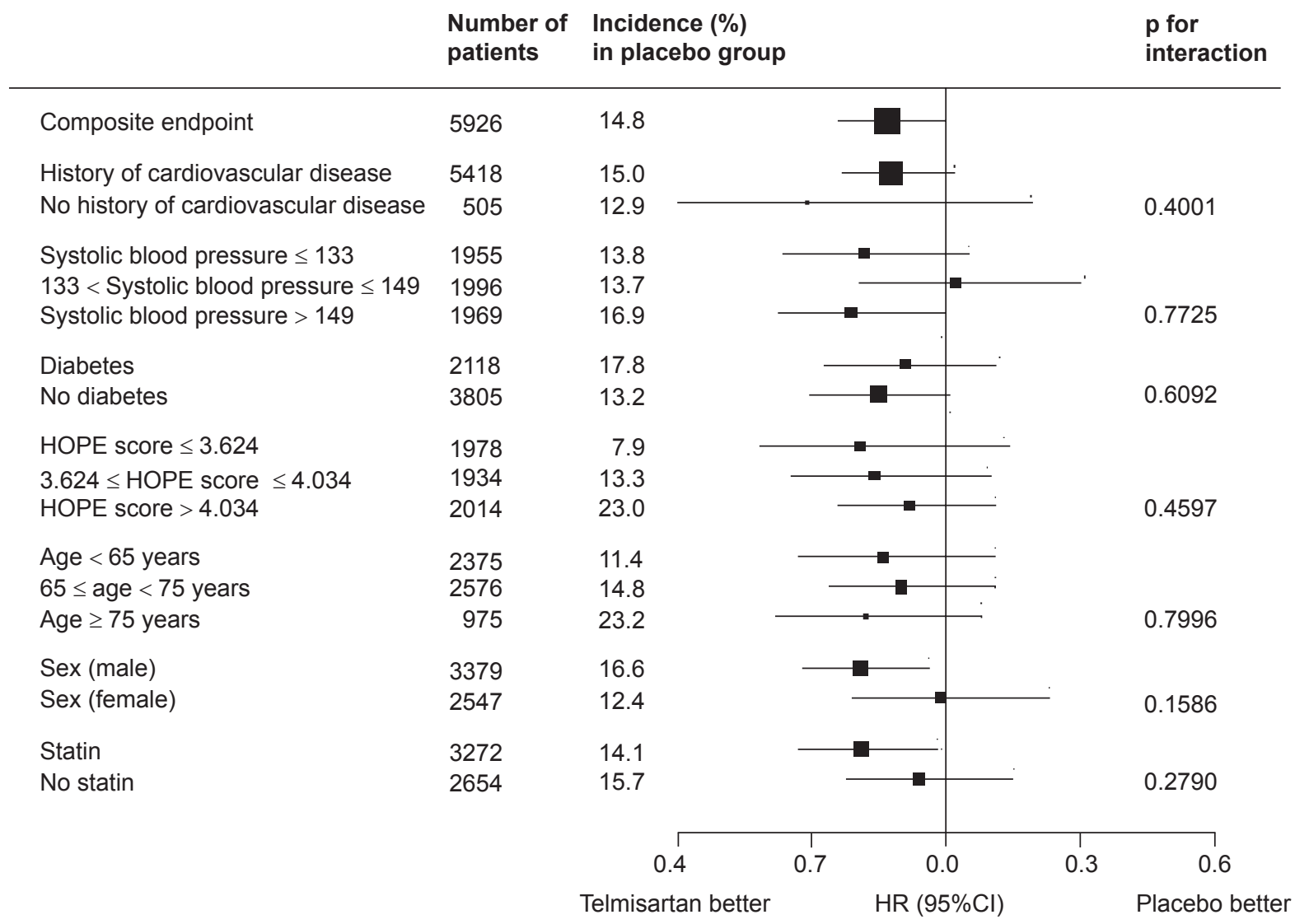

Figure 5 Relative risks for secondary HOPE study endpoint of cardiovascular death, MI and stroke in the TRANSCEND study. 
trial design as in VALIANT ${ }^{25}$ and ONTARGET, there was no additional benefit from the combination of ACEi and ARB beyond that of the single agent.

Combined ACEi and ARB treatment appears to reduce proteinuria more than the individual agents. Whether this is a blood pressure lowering effect is uncertain. The only evidence to show that combined ACEi and ARB treatment slows the progression of renal disease comes from the COOPERATE study; ${ }^{30}$ the results of which have recently been discredited and considered to be unreliable. ${ }^{31}$

\section{Was telmisartan better than placebo?}

The results of the TRANSCEND study showed that there was no significant difference in either the primary or secondary outcomes in patients receiving either telmisartan or placebo. The secondary HOPE endpoint of death/MI/stroke appeared to be reduced by telmisartan, yet the difference did not remain statistically significant after adjustments for multiple comparisons. Does this mean telmisartan is no better than placebo, despite being shown to retain almost all the vascular protective properties of ramipril in the ONTARGET study? There are several possible reasons for this apparent discrepancy. Firstly that the TRANSCEND study was underpowered, with too low event rates and an inadequate follow-up period to show an ambitious $19 \%$ relative risk reduction. For the secondary HOPE endpoint the event rate in the placebo group of the TRANSCEND study was 3.2\%/ year but in the HOPE study the event rate was $4.4 \%$ / year. Yet in the EUROPA study, perindopril did show a $20 \%$ reduction of the combined endpoint of cardiovascular death/MI and cardiac arrest with lower event rates than were observed in the HOPE study and over a 4.2-year observation period.

A second possible explanation is the legacy effect of pre-treatment with either ACEi or ARB. The design and baseline characteristics paper $^{27}$ indicates that $58.1 \%$ of patients were receiving an ACEi and 29.9\% an ARB at the time of randomization. In the HOPE-TOO study, ${ }^{32}$ there were persistent benefits from the 5-year period of ramipril treatment, that remained for at least 3 (median 2.6 years) after the randomization period ended. A similar persistent legacy of treatment was observed in the AIREX study of post-myocardial heart failure. ${ }^{33}$ Hence it is possible that the benefits of telmisartan were reduced because of the persistent legacy of pre-treatment with either ACEi or ARB in the placebo group.

\section{Application of ONTARGET and TRANSCEND}

The results of these two important trials indicate that for patients at high risk for vascular events, either ramipril or telmisartan can be used to reduce event rates. Whether a physician uses an ACEi or ARB for vascular protection will depend upon many factors, including habit, cost and adverse effects of treatment. Yet telmisartan was shown to be safe in ACEi intolerant patients and from the ONTARGET study preserved almost all the vasculoprotective benefits of ramipril. Telmisartan was well tolerated with less patients discontinuing treatment due to cough (telmisartan 93 vs ramipril 360) or angio-edema (10 vs 25). Although hypotension was more frequent with telmisartan (229 vs 149), the incidence of syncope was very similar (19 vs 15). Therefore it would not be unreasonable, despite the results of TRANSCEND, to recommend ARB usage in ACEi intolerant individuals. Guidelines have started to recommend ACEi and ARBs as alternative choices for vascular protection especially in high risk individuals with diabetes. ${ }^{34}$

\section{Disclosures}

The author has no conflicts of interest to disclose.

\section{References}

1. Wagenaar LJ, Voors AA, Buikema H, van Gilst WH. Angiotensin receptors in the cardiovascular system. Can J Cardiol. 2002;18(1331)1339.

2. Yusuf S, Teo KK, Pogue J, Dyal L, Copland I, Schumacher H, Dagenais G, Sleight P, Anderson C. Telmisartan, ramipril, or both in patients at high risk for vascular events. $N$ Engl J Med. 2008;358(15):1547-1559.

3. The Telmisartan Randomised AssessmeNt Study in ACE iNtolerant subjects with cardiovascular Disease (TRANSCEND) Investigators. Effects of the angiotensin-receptor blocker telmisartan on cardiovascular events in high-risk patients intolerant to angiotensinconverting enzyme inhibitors: a randomised controlled trial. Lancet. Published on-line 29 August 2008, DOI:10.1016/S01406736(08)61242-1248.

4. Dzau VJ. Mechanism of protective effects of ACE inhibition on coronary artery disease. Eur Heart J. 1998;19 Suppl J:J2-J6.

5. Brown NJ, Kumar S, Painter CA, Vaughan DE. ACE inhibition versus angiotensin type 1 receptor antagonism: differential effects on PAI-1 over time. Hypertension. 2002;40(6):859-865.

6. Kim MP, Zhou M, Wahl LM. Angiotensin II increases human monocyte matrix metalloproteinase-1 through the AT2 receptor and prostaglandin E2: Implications for atherosclerotic plaque rupture. J Leukoc Biol. 2005; 78:195-201.

7. The SOLVD investigators. Effect of enalapril on survival in patients with reduced ejection fraction and congestive heart failure. $N$ Engl J Med. 1991;325:293-302.

8. Pfeffer MA, Braunwald E, Moye LA, et al. Effect of captopril on mortality and morbidity in patients with left ventricular dysfunction after myocardial infarction. Results of the survival and ventricular enlargement trial. The SAVE Investigators. $N$ Engl $J$ Med. 1992;327(10):669-677.

9. Rutherford JD, Pfeffer MA, Moye LA, et al. Effects of captopril on ischemic events after myocardial infarction. Results of the Survival and Ventricular Enlargement trial. SAVE Investigators. Circulation. 1994;90(4):1731-1738.

10. Yusuf S, Sleight P, Pogue J, Bosch J, Davies R, Dagenais G. Effects of an angiotensin-converting-enzyme inhibitor, ramipril, on cardiovascular events in high-risk patients. The Heart Outcomes Prevention Evaluation Study Investigators (see comments). $N$ Engl $J$ Med. 2000;342(3):145-153. 
11. The EURopean trial On reduction of cardiac events with Perindopril in stable coronary Artery disease Investigators. Efficacy of perindopril in reduction of cardiovascular events among patients with stable coronary artery disease: randomised, double-blind, placebo-controlled, multicentre trial (the EUROPA study). Lancet. 2003;362:782-788.

12. The PEACE Investigators. Angiotensin-Converting-Enzyme Inhibitionin Stable Coronary Artery Disease. N Engl J Med. 2004;351:2058-2068.

13. Dagenais GR, Pogue J, Fox K, Simoons ML, Yusuf S. Angiotensinconverting-enzyme inhibitors in stable vascular disease without left ventricular systolic dysfunction or heart failure: a combined analysis of three trials. Lancet. 2006;368(9535):581-588.

14. Danchin N, Cucherat M, Thuillez C, Durand E, Kadri Z, Steg PG. Angiotensin-converting enzyme inhibitors in patients with coronary artery disease and absence of heart failure or left ventricular systolic dysfunction: an overview of long-term randomized controlled trials. Arch Intern Med. 2006;166(7):787-796.

15. Granger CB, Ertl G, Kuch J, et al. Randomized trial of candesartan cilexetil in the treatment of patients with congestive heart failure and a history of intolerance to angiotensin-converting enzyme inhibitors. Am Heart J. 2000;139(4):609-617.

16. Granger CB, McMurray JJ, Yusuf S, et al. Effects of candesartan in patients with chronic heart failure and reduced left-ventricular systolic function intolerant to angiotensin-converting-enzyme inhibitors: the CHARM-Alternative trial. Lancet. 2003;362(9386):772-776.

17. Brenner BM, Cooper ME, de Z, et al. Effects of losartan on renal and cardiovascular outcomes in patients with type 2 diabetes and nephropathy. N Engl J Med. 2001;345(12):861-869.

18. Parving HH, Lehnert H, Brochner M, Gomis R, Andersen S, Arner P. The effect of irbesartan on the development of diabetic nephropathy in patients with type 2 diabetes. N Engl J Med. 2001;345(12):870-878.

19. Pfeffer MA, Swedberg K, Granger CB, et al. Effects of candesartan on mortality and morbidity in patients with chronic heart failure: the CHARM-Overall programme. Lancet. 2003;362(9386):759-766.

20. McMurray JJ, Ostergren J, Swedberg K, et al. Effects of candesartan in patients with chronic heart failure and reduced left-ventricular systolic function taking angiotensin-converting-enzyme inhibitors: the CHARM-Added trial. Lancet. 2003;362(9386):767-771.

21. Demers C, McMurray JJ, Svedberg K, et al. Impact of candesartan in preventing myocardial infarction: results of the Candesartan in Heart failure Assessment of Reduction in Mortality and Morbidity (CHARM) Programme. Circulation. 110 2004;Suppl III:III-514.

22. Dahlof B, Devereux RB, Kjeldsen SE, et al. Cardiovascular morbidity and mortality in the Losartan Intervention For Endpoint reduction in hypertension study (LIFE): a randomised trial against atenolol. Lancet. 2002;359(9311):995-1003.
23. Julius S, Kjeldsen SE, Weber M, et al. Outcomes in patients at high cardiovascular risk treated with regimens based on valsartan or amlodipine: the VALUE randomised trial. Lancet. 2004;363:2022-2031.

24. Flather MD, Yusuf S, Kober L, et al. Long-term ACE-inhibitor therapy in patients with heart failure or left-ventricular dysfunction: a systematic overview of data from individual patients. ACE-Inhibitor Myocardial Infarction Collaborative Group (see comments). Lancet. 2000;355(9215):1575-1581.

25. Pfeffer MA, McMurray JJ, Velazquez EJ, et al. Valsartan, captopril, or both in myocardial infarction complicated by heart failure, left ventricular dysfunction, or both. $N$ Engl $J$ Med. 2003;349(20):1893-1906

26. Strauss MH, Hall AS. Angiotensin receptor blockers may increase risk of myocardial infarction: unraveling the ARB-MI paradox. Circulation. 2006;114(8):838-854.

27. Teo KK, Burton JR, Buller C, Plante S, Yokoyama S, Montague TJ. Rationale and design features of a clinical trial examining the effects of cholesterol lowering and angiotensin-converting enzyme inhibition on coronary atherosclerosis: Simvastatin/Enalapril Coronary Atherosclerosis Trial (SCAT). SCAT Investigators. Can J Cardiol. 1997;13(6):591-599.

28. Mann JF, Schmieder RE, McQueen M, et al. Renal outcomes with telmisartan, ramipril, or both, in people at high vascular risk (the ONTARGET study): a multicentre, randomised, double-blind, controlled trial. Lancet. 2008;372(9638):547-553.

29. Yusuf S, Diener HC, Sacco RL, et al. Telmisartan to prevent recurrent stroke and cardiovascular events. N Engl J Med. 2008.

30. Nakao N, Yoshimura A, Morita H, Takada M, Kayano T, Ideura T. Combination treatment of angiotensin-II receptor blocker and angiotensinconverting-enzyme inhibitor in non-diabetic renal disease (COOPERATE): a randomised controlled trial. Lancet. 2003;361(9352):117-124.

31. Kunz R, Wolbers M, Glass T, Mann J. The COOPERATE trial: a letter of concern. Lancet. 2008;371:1575-1576.

32. HOPE/HOPE-TOO Study Investigators. Results of the HOPE study extension. long-term effects of ramipril on cardiovascular events and on diabetes. Circulation. 2005;112:1339-1346.

33. Hall AS, Murray GD, Ball SG. Follow-up study of patients randomly allocated ramipril or placebo for heart failure after acute myocardial infarction: AIRE Extension (AIREX) Study. Lancet. 1997;349:1493-1497.

34. Canadian Diabetes Association Clinical Practice Guidelines Expert Committee. Canadian Diabetes Association 2008 clinical practice guidelines for the prevention and management of diabetes in Canada. Can J Diabetes. 2008;32 suppl 1:S1-S201. 
